\title{
Unilateral and Equitransitive Tilings by Squares
}

\author{
D. Schattschneider \\ Mathematics Department, Moravian College, \\ Bethlehem, PA 18018, USA \\ schattdo@moravian.edu
}

\begin{abstract}
Recent results obtained by Martini et al. [4] facilitate the proof that there are exactly eight unilateral and equitransitive tilings of the plane by squares of three sizes. This refutes a conjecture by Schattschneider that appears in the book Tilings and Patterns by Grünbaum and Shephard [2].
\end{abstract}

\section{Introduction and Background}

A problem posed by Grünbaum and Shephard led me in 1978 to consider unilateral and equitransitive tilings by squares of three sizes. Using the Conway Criterion [5], I showed there are (up to equivalence) precisely five such tilings of type p2, and I conjectured there were no more (see Fig. 1). In their 1987 book, the five tilings are displayed and an exercise asks the reader to prove this conjecture [2, p. 81]. However, in 1997, the conjecture was shown to be false by Martini et al. [4], who discovered such a tiling of type pgg (see Fig. 2(a)), and shortly thereafter Grünbaum produced two more of type pgg (see Fig. 2(b),(c)). Here we use the results of [4] to show that the list is now complete: There are precisely eight unilateral and equitransitive tilings by squares of three sizes.

First, some definitions. "A tiling by regular polygons is equitransitive if each set of mutually congruent tiles forms one transitivity class ... [and is] unilateral [if] each edge of the tiling is a side of at most one polygon" [2, pp. 70 and 73]. Thus in a unilateral tiling, no two congruent tiles can have a common side, and in an equitransitive tiling, for any pair of congruent tiles, there is a symmetry of the tiling that maps one tile onto the other. For purposes of classification, two tilings $\mathcal{T}$ and $\mathcal{T}^{\prime}$ will be considered equivalent if they are homeomeric (topologically equivariant). That is, if $G$ and $G^{\prime}$ are the symmetry groups of $\mathcal{T}$ and $\mathcal{T}^{\prime}$, then there exists a homeomorphism $\varphi$ that maps $\mathcal{T}$ onto $\mathcal{T}^{\prime}$ (preserving tiles, edges, and vertices of the tiling) such that $\varphi G \varphi^{-1}=G^{\prime}$. In the discussion that follows, we use the acronym UET3 for "unilateral and equitransitive tiling by squares of three sizes." 


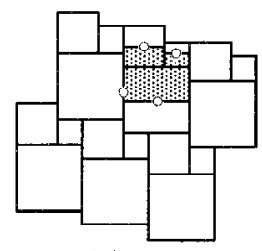

(a)

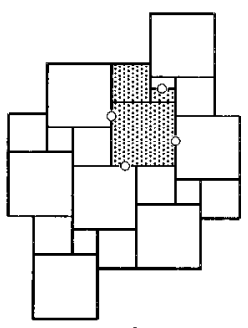

(b)

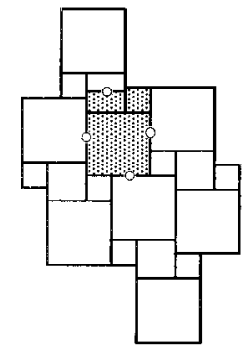

(c)

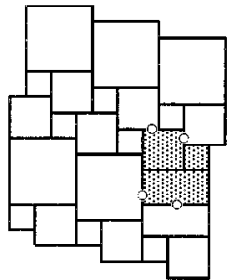

(d)

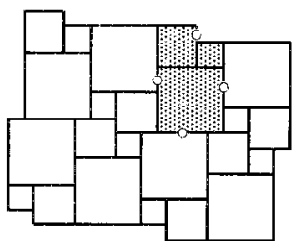

(e)

Fig. 1. The five unilateral and equitransitive p2 tilings by squares of three sizes. Fundamental domains are shaded; circles denote twofold centers of rotation.

In order to state the results in [4], we first need some definitions. Let $\mathcal{T}$ be a tiling of the plane by squares. A tile $T$ of $\mathcal{T}$ is a vortex if, when $T$ is given an orientation, each side of $T$ may be extended along an edge of the tiling according to that orientation, but may not be extended in the opposite direction. A vortex tile is shown at the top of the next page; note that every vertex of $T$ belongs to the relative interior of a side of another tile.

A corona of a single tile is the set of all tiles in $\mathcal{T}$ that have nonempty intersection with the tile. (In [4] a corona is called a neighborly environment.)

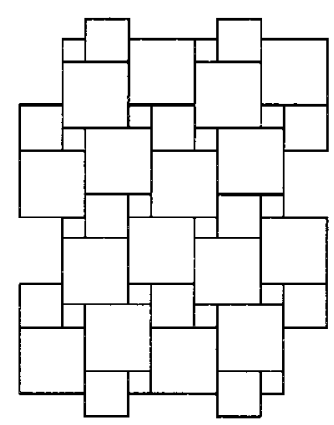

(a)

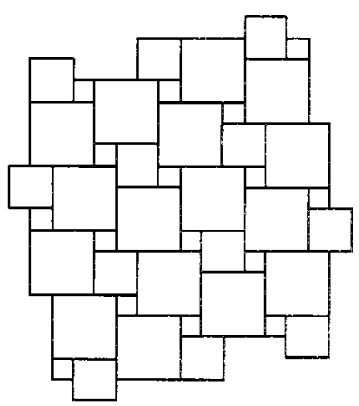

(b)

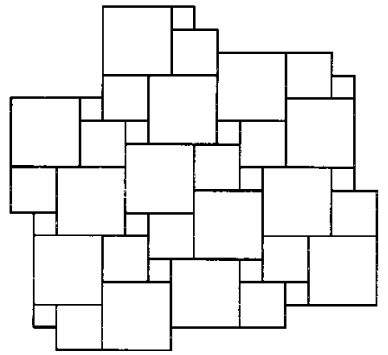

(c)

Fig. 2. The three unilateral and equitransitive pgg tilings by squares of three sizes. Edges of squares are in the ratio $1: 2: 3$. In (a) glide-reflection axes are parallel to the edges of the squares; in (b) and (c) glide-reflection axes are parallel to diagonals of the squares. 


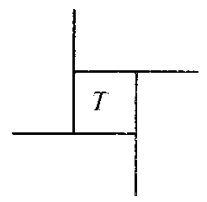

Now let $\mathcal{T}$ be a unilateral tiling of the plane by squares of at least three distinct edgelengths and let $\lambda_{1}<\lambda_{2}<\lambda_{3}$ be the three smallest edge-lengths. In [4] the authors prove the following:

(1) Every $\lambda_{1}$-tile and every $\lambda_{2}$-tile of $\mathcal{T}$ is a vortex.

(2) At least one $\lambda_{1}$-tile has a common point with a tile of size $\lambda_{2}$ and a tile of size $\lambda_{3}$.

When $\mathcal{T}$ is a unilateral tiling by exactly three squares of sizes $\lambda_{1}<\lambda_{2}<\lambda_{3}$, the authors also prove:

(3) There is a $\lambda_{3}$-tile in $\mathcal{T}$ that is not a vortex.

(4) $\lambda_{1}+\lambda_{2}=\lambda_{3}$.

(5) No $\lambda_{3}$-tile has a point in common with two distinct $\lambda_{2}$-tiles.

Finally, the authors obtain all theoretically possible coronas that can occur in a unilateral tiling $\mathcal{T}$ with squares of three sizes (Theorems 4 and 5 in [4]). When $\mathcal{T}$ is also equitransitive, condition (2) implies that every corona of every tile must contain tiles of all three sizes. Thus we can display all theoretically possible coronas that can occur in a UET3 (we do not distinguish between a corona and its reflection). These are displayed in Figs. 3-6.

Each tile of size $\lambda_{i}$ in Figs. 3-6 has been labeled with the number $i$, and under each corona there is a sequence that is a signature for that corona. The signature is determined as follows: Every corona contains an L-shape block of three squares of different sizes: size $\lambda_{3}$ (at the bottom of the block), size $\lambda_{2}$ (at the upper left of the block), and size $\lambda_{1}$ (at the upper right of the block). Beginning with the tile labeled with a $*$ that abuts the top of the $\lambda_{1}$ tile of this block (or abuts the right side of the $\lambda_{1}$ tile if no tile abuts its top), the sizes of the tiles that surround the central tile are listed in clockwise cyclic order. These signatures are unique, since the only coronas that contain more than one of these L-shape blocks have half-turn symmetry, and hence have symmetric signatures.

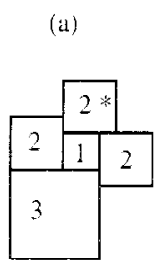

2. 2. 3, 2

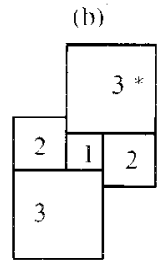

$3.2,3,2$

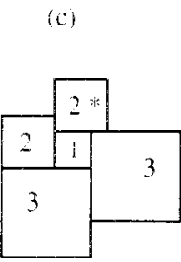

2.3. 3.2 2

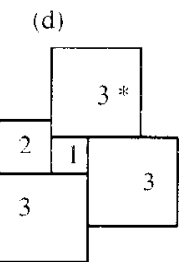

$3,3,3,2$

Fig. 3. All possible $\lambda_{1}$-coronas with signatures of surrounding squares. 
(a)

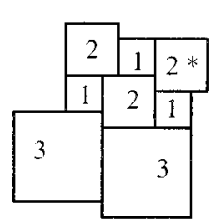

$2,1,3,3,1,2,1$ (b)

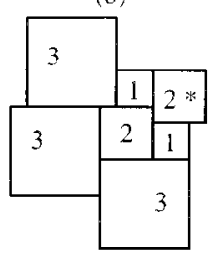

$2,1,3,3,3,1$ (c)

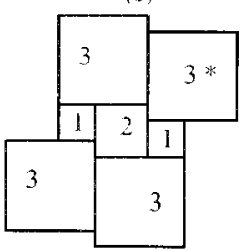

3. 1, 3, 3, 1, 3 (d)

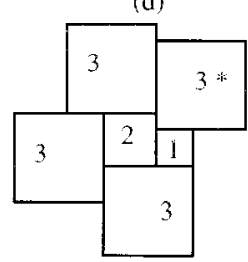

$3,1,3,3,3$

Fig. 4. All possible $\lambda_{2}$-coronas with signatures of surrounding squares.

(a)

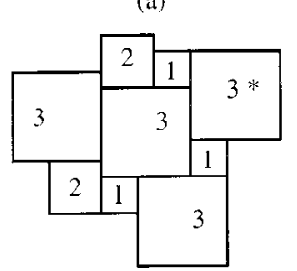

$3,1,3,1,2,3,2,1$

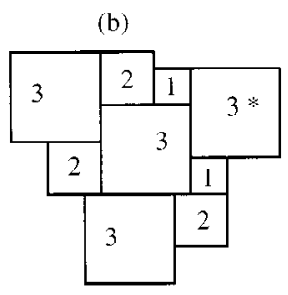

$3,1,2,3,2,3,2,1$

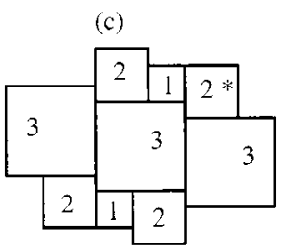

$2,3,2,1,2,3,2,1$ (d)

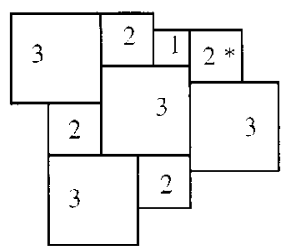

$2,3,2,3,2,3,2,1$

Fig. 5. All possible $\lambda_{3}$-coronas in which $\lambda_{2} \neq 2 \lambda_{1}$ may occur, with signatures of surrounding squares.

(a)

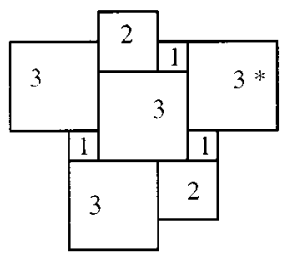

3. $1,2,3,1,3,2,1$

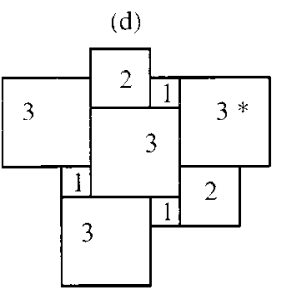

$3,2,1,3,1,3,2,1$ (b)

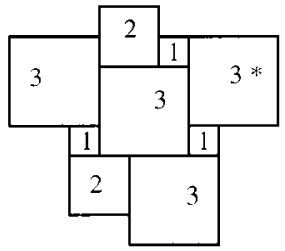

$3,1,3,2,1,3,2,1$

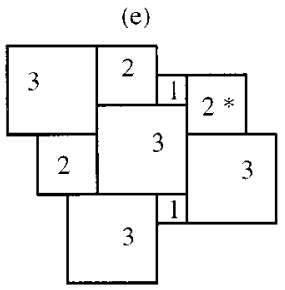

$2,3,1,3,2,3,2,1$ (c)

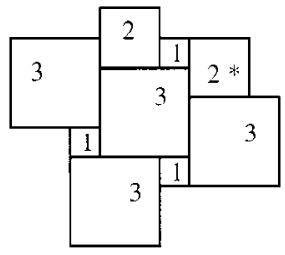

$2,3,1,3,1,3,2,1$

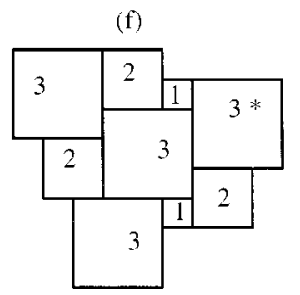

$3,2,1,3,2,3,2,1$

Fig. 6. All possible $\lambda_{3}$-coronas in which $\lambda_{2}=2 \lambda_{1}$, with signatures of surrounding squares. 


\section{The Proof}

In the proof of the theorem, we test to see which of the coronas in Figs. 3-6 are actually realizable in a UET3.

Theorem. There are exactly eight unilateral and equitransitive tilings by squares of three different sizes.

Proof. Suppose that $\mathcal{T}$ is a UET3. Since $\mathcal{T}$ is unilateral, $\mathcal{T}$ must satisfy conditions (1)(5) given earlier. Since $\mathcal{T}$ is equitransitive, all coronas of tiles of size $\lambda_{i}$ are congruent for $i=1,2,3$. Thus $\mathcal{T}$ 's $\lambda_{1}$-coronas are equivalent to one of the four types in Fig. 3, its $\lambda_{2}$-coronas to one of the four types in Fig. 4 , and its $\lambda_{3}$-coronas to one of the ten types in Figs. 5 and 6. We need to test which combinations of $\lambda_{1^{-}}, \lambda_{2^{-}}$, and $\lambda_{3}$-coronas are actually realizable in a single UET3. To indicate how this is done, we go through the argument for one case.

Suppose that $\mathcal{T}$ has a $\lambda_{1}$-corona as shown in Fig. 3(a); this has signature 2, 2, 3, 2. Observing the way in which tiles in this corona surround each of its $\lambda_{2}$-tiles, we see that the $\lambda_{2}$-coronas in $\mathcal{T}$ must have signatures that contain the partial sequences $2,1,2$ and 3, 1, 2, and $2,1,3$. The only $\lambda_{2}$-corona with such a signature occurs in Fig. 4(a), with signature $2,1,3,3,1,2,1$ and this corona has all partial $\lambda_{1}$-signatures in agreement with the signature $2,2,3,2$. Next we examine the partial $\lambda_{3}$-coronas in both figures and find that the $\lambda_{3}$-coronas in $\mathcal{T}$ must have signatures that contain the partial sequences $2,1,2$ (from Fig. 3(a)), 1, 2, 3 and 3, 2, 1 (from Fig. 4(a)). We find that only the coronas in Figs. 5(c),(d) and $6(\mathrm{c}),(\mathrm{e})$ have signatures that contain these partial sequences. Checking the partial $\lambda_{1}$ - and $\lambda_{2}$-coronas in each of these figures shows that only Fig. 5(c) is compatible with the $\lambda_{1}$-signature 2, 2, 3, 2 and $\lambda_{2}$-signature 2, 1, 3, 3, 1, 2, 1. (Figures 6(c) and (e) have partial $\lambda_{1}$-signatures $3,3,3$, and Fig. 5(d) has partial $\lambda_{2}$-signature $3,3,3$.) The tiling shown in Fig. 1(d) has all three of the required coronas. We then check that there is no other way that these three coronas can occur simultaneously in a unilateral and equitransitive tiling. To do this, we check that, beginning with the given $\lambda_{1}$-corona, its partial $\lambda_{2}$-coronas can be completed in only one way to have signature $2,1,3,3,1,2,1$. Then in this larger patch, we check that the partial $\lambda_{3}$-coronas completely determine the full $\lambda_{3}$-coronas with the desired signature $2,3,2,1,2,3,2,1$. The transitivity of the symmetry group of the tiling on coronas then completes the tiling uniquely; hence this is the only tiling that has a $\lambda_{1}$-corona with signature $2,2,3,2$.

Each of the other possibilities is deduced in a similar manner. Table 1 summarizes the process of determining which combinations of $\lambda_{1^{-}}, \lambda_{2^{-}}$, and $\lambda_{3}$-coronas are simultaneously possible in a unilateral and equitransitive tiling. Each row of the table should be read left-to-right; "compatibility" of a corona means that it is compatible with all the information that precedes it on the left. There are nine possible combinations that are simultaneously possible, and eight of these are realizable, corresponding to the eight tilings in Figs. 1 and 2. We now show that the ninth combination, the coronas in Figs. 3(d), 4(c), and 6(a), cannot occur in an equitransitive tiling.

Suppose there is a tiling with these three signatures for its coronas. Then we can build up a patch of the tiling; refer to Fig. 7. Begin with the 3, 3, 3, 2 corona about the 
Table 1. The coronas that may be simultaneously possible in a UTE3.

\begin{tabular}{|c|c|c|c|c|c|c|}
\hline$\lambda_{1}$-Corona & $\begin{array}{c}\text { Partial } \\
\lambda_{2} \text {-coronas }\end{array}$ & $\begin{array}{c}\text { Partial } \\
\lambda_{3} \text {-coronas }\end{array}$ & $\begin{array}{l}\text { Compatible } \\
\lambda_{2} \text {-coronas }\end{array}$ & $\begin{array}{c}\text { Partial } \\
\lambda_{3} \text {-coronas }\end{array}$ & $\begin{array}{l}\text { Compatible } \\
\lambda_{2} \text {-coronas }\end{array}$ & Tiling \\
\hline $2,2,3,2$ & $3,1,2$ & $2,1,2$ & $2,1,3,3,1,2,1$ & $1,2,3$ & $2,3,2,1,2,3,2,1$ & Fig. 1(d) \\
\hline \multirow[t]{2}{*}{ Fig. 3(a) } & $2,1,2$ & & Fig. 4(a) & $3,2,1$ & Fig. 5(c) & \\
\hline & $2,1,3$ & & & & & \\
\hline \multirow{5}{*}{$\begin{array}{l}3,2,3,2 \\
\text { Fig. 3(b) }\end{array}$} & $3,1,3$ & $2,1,2$ & $3,1,3,3,1,3$ & $1,2,3$ & $2,3,2,1,2,3,2,1$ & Fig. 1(a) \\
\hline & & & Fig. 4(c) & $3,2,1$ & Fig. 5(c) & \\
\hline & & & $3,1,3,3,3$ & $3,2,3$ & $2,3,2,3,2,3,2,1$ & Fig. 1(b) \\
\hline & & & Fig. 4(d) & $1,2,3$ & Fig. $5(\mathrm{~d})$ & \\
\hline & & & & $3,2,1$ & & \\
\hline $2,3,3,2$ & $3,1,2$ & $3,1,2$ & $2,1,3,3,3,1$ & $1,2,3$ & $3,1,2,3,2,3,2,1$ & Fig. 1(e) \\
\hline \multirow[t]{2}{*}{ Fig. 3(c) } & $2,1,3$ & $2,1,3$ & Fig. 4(b) & $3,2,3$ & Fig. $5(\mathrm{~b})$ & \\
\hline & & & & $3,2,1$ & & \\
\hline
\end{tabular}

3, 2, 1, 3, 2, 3, 2, $1 \quad$ Fig. 2(c)

Fig. 6(f)

\begin{tabular}{lcccccc}
\hline 3, 3, 3,2 & $3,1,3$ & $3,1,3$ & $3,1,3,3,1,3$ & $1,2,3$ & $3,1,3,1,2,3,2,1$ & Fig. 1(c) \\
Fig. 3(d) & & $2,1,3$ & Fig. 4(c) & $3,2,1$ & Fig. 5(a) & \\
& & $3,1,2$ & & & &
\end{tabular}

$3,1,3,2,1,3,2,1 \quad$ Fig. 2(b) Fig. 6(b)

3, 2, 1, 3, 1, 3, 2, $1 \quad$ Fig. 2(a) Fig. 6(d)

$3,1,2,3,1,3,2,1$

Fig. 6(a)

\begin{tabular}{cll}
\hline $3,1,3,3,3$ & $3,2,3 \quad$ None \\
Fig. 4(d) & $3,2,1$ \\
& $1,2,3$ & \\
\hline
\end{tabular}

black $\lambda_{1}$-tile. Then complete the $3,1,3,3,1,3$ corona about the $\lambda_{2}$-tile labeled 2 , and then the $3,1,2,3,1,3,2,1$ corona about the $\lambda_{3}$-tile labeled 3 . The placement of tiles in each of these successive coronas is completely determined by its partial corona and the specified corona sequence. However, then the corona of the $\lambda_{3}$-tile labeled $\mathrm{X}$ contains the partial sequence $3,1,3,1$, a contradiction. Thus there is no equitransitive tiling with the specified coronas.

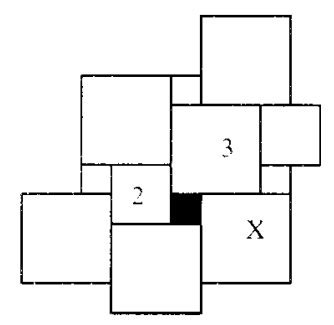

Fig. 7 
Remarks. 1. Bölcskei, using the Poincaré method of examining all possible fundamental domains for such a tiling (as in [3]) has provided a different proof of the theorem [1].

2. There are several partial results on the corresponding problem of unilateral and equitransitive tilings by four squares of different sizes, but they are far from complete. As with UET3 problem, there are p2 and pgg tilings (more than 30), and special cases occur when the squares have edges in the ratios $1: 2: 3: 4$. Unlike the UET3 problem, there are nonsimple tilings in the case of four squares.

\section{Acknowledgment}

I want to express my warm thanks to Branko Grünbaum for his awakening my interest in the subject of tilings, and for his enduring encouragement of my work over the years.

\section{References}

1. A. Bölcskei, Classification of unilateral and equitransitive tilings by squares of three sizes, Beiträge Algebra Geom. 41(1) (2000), 267-277.

2. B. Grünbaum and G. C. Shephard, Tilings and Patterns, Freeman, San Francisco, CA, 1987.

3. Z. Lǔcíc and E. Molnár, Combinatorial classification of fundamental domains of finite area for planar discontinuous isometry groups, Arch. Math. 54 (1990), 511-520.

4. H. Martini, E. Makai, and V. Soltan, Unilateral tilings of the plane with squares of three sizes, Beiträge Algebra Geom. 39(2) (1998), 481-495.

5. D. Schattschneider, Will it tile? Try the Conway Criterion!, Math. Mag. 53 (1980), 224-233.

Received January 6, 1999, and in revised form October 2, 1999. Online publication May 16, 2000. 